\title{
Interpersonal Violence in Alcohol Complicated Marital Relationships (A Study from India)
}

\author{
Selwyn Stanley
}

Published online: 21 October 2008

(C) Springer Science + Business Media, LLC 2008

Erratum to: J Fam Viol (2008) 23:767-776

DOI 10.1007/s10896-008-9202-7

The original version of this article unfortunately contained a mistake. The presentation of Table 3 was incorrect, the 'Mean squares' and ' $F$ value' columns under the last column for 'Danger assessment' were missing. The corrected table is given below.

Table 3 ANOVA summary table for both groups based on subject dimensions and background factors

\begin{tabular}{|c|c|c|c|c|c|c|c|c|c|c|}
\hline \multirow[t]{3}{*}{ Variables } & & \multicolumn{9}{|c|}{ Subject dimensions } \\
\hline & & \multicolumn{3}{|c|}{ Overall conflict } & \multicolumn{3}{|c|}{ Comm. apprehension } & \multicolumn{3}{|c|}{ Danger assessment } \\
\hline & & $\begin{array}{l}\text { Sum of } \\
\text { squares }\end{array}$ & $\begin{array}{l}\text { Mean } \\
\text { squares }\end{array}$ & $F$ Value & $\begin{array}{l}\text { Sum of } \\
\text { squares }\end{array}$ & $\begin{array}{l}\text { Mean } \\
\text { squares }\end{array}$ & $F$ Value & $\begin{array}{l}\text { Sum of } \\
\text { squares }\end{array}$ & $\begin{array}{l}\text { Mean } \\
\text { squares }\end{array}$ & $F$ Value \\
\hline \multirow{2}{*}{$\begin{array}{l}\text { Consanguinity } \\
\text { (present/absent) }\end{array}$} & $\mathrm{BG}^{\mathrm{a}}$ & $63,730.89$ & $21,243.63$ & $84.82, p<0.001$ & 882.96 & 294.32 & $8.49, p<0.001$ & $2,431.52$ & 810.51 & $76.06, p<0.001$ \\
\hline & $\mathrm{WG}^{\mathrm{b}}$ & $36,568.95$ & 250.47 & & $5,062.84$ & 34.68 & & $1,555.74$ & 10.66 & \\
\hline \multirow{2}{*}{$\begin{array}{l}\text { Occupation } \\
\text { (employed/ } \\
\text { housewife) }\end{array}$} & $\mathrm{BG}^{\mathrm{a}}$ & $63,915.79$ & $21,305.26$ & $85.49, p<0.001$ & $1,037.10$ & 345.701 & $10.28, p<0.001$ & $2,422.11$ & 807.37 & $75.31, p<0.001$ \\
\hline & $\mathrm{WG}^{\mathrm{b}}$ & $36,384.04$ & 249.20 & & $4,908.68$ & 33.621 & & $1,565.14$ & 10.72 & \\
\hline \multirow{2}{*}{$\begin{array}{l}\text { Type of family } \\
\text { (nuclear/joint) }\end{array}$} & $\mathrm{BG}^{\mathrm{a}}$ & $63,938.84$ & $21,312.94$ & $85.57, p<0.001$ & 866.26 & 288.75 & $8.30, p<0.001$ & $2,425.96$ & 808.65 & $75.61, p<0.001$ \\
\hline & $\mathrm{WG}^{\mathrm{b}}$ & $36,360.99$ & 249.04 & & $5,079.53$ & 34.79 & & $1,561.29$ & 10.69 & \\
\hline \multirow{2}{*}{$\begin{array}{l}\text { Type of marriage } \\
\text { (love/arranged) }\end{array}$} & $\mathrm{BG}^{\mathrm{a}}$ & $63,730.89$ & $21,243.63$ & $84.81, p<0.001$ & $5,062.83$ & 294.31 & $8.48, p<0.001$ & $2,431.51$ & 810.50 & $76.06, p<0.001$ \\
\hline & $\mathrm{WG}^{\mathrm{b}}$ & $36,568.95$ & 250.47 & & $5,945.79$ & 34.67 & & $1,555.74$ & 10.65 & \\
\hline
\end{tabular}

${ }^{\text {a }}$ Between groups $d f=3$

${ }^{\mathrm{b}}$ Within groups $d f=146$

The online version of the original article can be found at http://dx.doi. org/10.1007/s10896-008-9202-7.

S. Stanley $(\bowtie)$

Faculty of Health and Social Work,

School of Psychosocial Studies, University of Plymouth,

Plymouth PL4 8AA, UK

e-mail: Selwyn.stanley@plymouth.ac.uk 